International Journal of Distributed and Parallel Systems (IJDPS) Vol.3, No.1, January 2012

\title{
AN IMPROVED PERFormanCE ANALYSIS OF PRIORITY SCHEDULING ALGORITHM IN MODIFIED AD HOC GRID LAYER
}

\author{
R. Bhaskaran ${ }^{1}$ and V.Parthasarathy ${ }^{2}$ \\ ${ }^{1}$ Department of Information Technology, PSNA College of Engg. and Technology, \\ Dindigul, Tamilnadu, India \\ bhaskaranmdu@gmail.com \\ ${ }^{2}$ Department of Computer Science and Engineering, Chettinad College of of Engg. and \\ Technology, Karur, Tamilnadu, India \\ sarathy.vp@gmail.com
}

\section{ABSTRACT}

An improved performance analysis of priority based scheduling algorithm for the ad hoc grid layer is presented in this paper. A priority queuing model is developed to reduce the execution time of the jobs. Here, jobs to be dispatched based on priority. Execution time is one prime metric proposed for the system. We have achieved the overall execution time for the proposed system for 25 jobs is 7.94 milliseconds whereas in the existing it is 26.725 milliseconds.

\section{KEYWORDS}

Modified ad hoc grid layer, wireless grid computing

\section{INTRODUCTION}

Grid computing is one of the developing computing initiatives that involve the aggregation of network connected computers to form a large scale distributed system for coordinated problem solving and resource sharing. In order to spread computing workload across the distributed system of computers, grid users can take advantage of enormous computational, storage and bandwidth resources, otherwise only be available within traditional multiprocessor supercomputers. Recent research shows that the mobile adhoc network applications are applied in the field of disaster management. In order to save the precious human lives ad hoc networks are used. The resource sharing potential of grid computing initiates the juncture of grid computing and mobile ad hoc environments. A Grnarov et al [1] developed grid computing implementations in adhoc networks. They investigated the adhoc network performances and tune the network grid parameters. And also they investigated the advantages and possible weakness of the model. They also provided the opportunities to make an optimization of the different parameters that influence the performance of the adhoc grid.

Zhoqun li et al [2] proposed light weight architecture with the integration of grid functionalities with ad hoc environment and find its application mainly in e- health care, finds the difficulty in managing priorities and a proposal of dropping low priority jobs during queue overflow. The primary issue addressed in the applications of mobile ad hoc grid is lack of application awareness.

Congestion control remains a critical issue cause by the saturation of network resources. Congestion is not a static resource problem but rather a dynamic resource allocation problem [3]. Ritha and Robert investigated a priority queuing model by using fuzzy set theory. It optimizes a fuzzy priority queuing models in which arrival rate, service rate are fuzzy parameters [4]. The DSW algorithm defines the membership functions of the performance 
measures of priority queuing system. Y Seok et al [5] discussed a queue management algorithm to utilize high data rate efficiently for the wireless LAN environment. Hey considered that the packets with short transmission time have higher priority. The fuzzy inference engine does not consider the priority which may be time consuming for real time applications. Hop-by-hop approach is followed in this model and each node forms a queue to its destination. Each node maintains a separate queue to its destination. Here also we consider the GDP approach adopted by Umut Akoyl [6] in conjunction with the key data-structure that is called as Per Destination Queue (PDQ). K.Kurowski et.al.,[7] in their work had followed hard and soft constraints to find a schedule that satisfies users preferences and they have used multi criteria approach for scheduling of jobs. The work follows advance reservation of resources for allocated jobs. Matthew smith et.al, [8] have introduced service oriented ad hoc grid, by providing computing resources on demand to every participant. Moreover they have addressed about the importance of ad hoc grid and its future expansion.

Carstesn Ernemann et.al.[9] had made a study of parallel job scheduling on single parallel machines following FCFS strategy. They have concluded that on submitting jobs with the requirement of large nodes may lead to poor quality of results on following the foresaid strategy.

Hameed vahdat [10] had developed a novel fuzzy algorithm for overall scheduling. The scheduling is a complex process and have focused on global scheduling dealing with clusters. They consider the communication ratio of the job. The authors have computed priority based on which scheduling is done. They have conferred that the job having maximum priority will be scheduled. B.Roy [11] has defined Scheduling as the process of identifying requirements, matching resources for computing jobs. Krzysztof et.al [12] have followed a multi criteria approach for scheduling jobs on the grid have presented two different models, as well as discussed about the advantages and applications of the practical applications. Gaurav Sharma et.al. [13] developed Congestion control and Scheduling algorithm, supporting single hop approach and guarantee the throughput in wireless networks.

Carole farad [14] has considered the problem of grid scheduling using tabu search using fuzzy rules. The developed scheduling algorithm aims to maximize the number of scheduled jobs. Tardiness is the technique followed for scheduling, which is not appropriate for real time applications. Alexander folling et.al [15] has addressed in his open research issues the usage of common performance metrics. First Come First Serve (FCFS) is the strategy used for incoming jobs at the local scheduler. Tarek helmy [15] had addressed Job scheduling as a major problem, since it is the fundamental and crucial step in achieving high performance. They have developed fuzzy c means algorithm with the goal to reduce the total execution time of jobs. The proposed method also provides the reduced execution time on comparison with existing methods.

In the proposed system, PDQ with M/M/1 queue model with single server with Poisson arrivals. The proposed system also introduces the queuing model followed by traffic engineering to avoid congestion. It also addresses the importance and performance of the prioritized jobs in AHGL and AHGL based congestion control.

The rest of the paper has been organized as follows. Proposed architecture for the job scheduling algorithm with dispatching in adhoc network is presented in Section 2. Section 3 discusses traffic engineering concepts for the proposed scheduling algorithm. In Section 4, parameters and measurement of performance metrics are discussed. Section 5 compares the results of non priority and priority based queuing system and its performance. Conclusion and further scope of the research are given in Section 6.

\section{ARCHITECTURE OF THE PROPOSED SYSTEM FOR JOB SCHEDULING AND DISPATCHING IN AD HOC NETWORKS}

The architecture of the proposed system is depicted in Figure 1. The categorization of jobs into high, medium and low priority jobs are arrived at the scheduler. The major functionalities of the 
scheduler is to dispatch the jobs to the queue in step 1 . The arrived packets based on the priority are scheduled to the PQ in step 2. It describes queuing functionality in ad hoc networks based on priority. In PQ the preference is given to high priority jobs, followed by medium and low priority jobs. The jobs sent from the scheduler are arrived at the dispatcher. In Step 3 the dispatched jobs are driven into ad hoc network. The association of wireless devices forming an ad hoc network, amongst the available nodes only the nodes with resources, capable of executing the deployed function forms the ad hoc network.

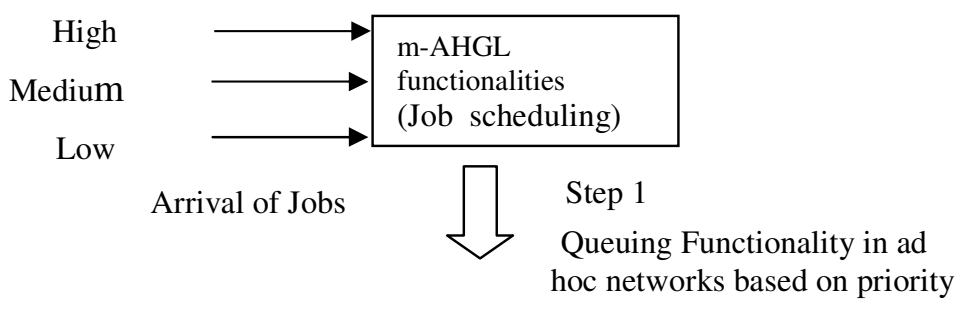

\begin{tabular}{|c|c|c|c|c|c|c|c|c|c|c|c|c|}
\hline$J_{H_{1}}$ & $J_{H_{2}}$ & & $J_{H_{n}}$ & $J_{M_{1}}$ & $J_{M_{2}}$ & - & $J_{M_{n}}$ & & $J_{L_{1}}$ & $J_{L_{2}}$ & - & $J_{L_{n}}$ \\
\hline $\mathrm{H}$ & $\mathrm{H}$ & & $\mathrm{H}$ & $\mathrm{M}$ & $\mathrm{M}$ & & $\mathrm{M}$ & & $\mathrm{L}$ & $\mathrm{L}$ & & $\mathrm{L}$ \\
\hline
\end{tabular}

Scheduling of jobs based on priority

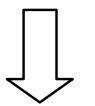

Step 2

Nodes participating to form ad hoc network

Step 3

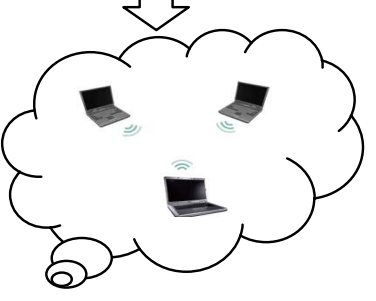

Fig 1. Proposed architecture for the Scheduling and dispatching of Jobs

\section{PRIORITY ALGORITHM FOR JOB SCHEDULING IN THE MOBILE AD HOC GRID LAYER}

In this section a modified priority algorithm for job scheduling in the mobile adhoc grid layer is presented in this section. The standard performance measures are average waiting time of job in a system, service time, waiting time in queue etc. The priority allocated is based on linguistic parameters using fuzzy scheme. Fuzzy based priority allocation consists of fuzzification, inference and defuzzification [16]. In the fuzzification step, fuzzy parameter values are converted to linguistic values (Low, Medium, High).

Main

Job $\rightarrow[1$ to $n]$

High priority job $\rightarrow[0-100]$ 
Medium priority job $\rightarrow$ 101-200]

Do

Low priority job $\rightarrow$ [201-300]

\{

Display "Start PQ" "Yes or No?"

Input Priority job

If Job Priority = "high"

Call Dispatcher Module

If Job Priority = "medium"

Check for presence of high priority jobs

\}

If yes call the high priority job

Else

\{

Call medium priority job

If Job Priority = "low"

Check for presence of high priority jobs

\}

If yes call the high priority job

Else

\{

Call medium priority job

If yes call medium priority job

\}

Else

\{

Call low priority job

If $\mathrm{PQ}=$ Empty

then

End

Make Dispatch

\{

job as integer [1 to $\mathrm{n}$ ]

Declare Job from scheduler as integer

The job on arriving from the scheduler

Display "The Job to be scheduled"

Input Priority Job

Display would you like to dispatch the job, "yes or no"?

\{

Check for high priority jobs in the queue

Display High priority jobs available, "yes or no"?

\}

If yes

\{

Display "Dispatching high priority job in the queue"

\}

Else

\{

Check for medium priority jobs in the queue

Display "Dispatching medium priority job in the queue"

\}

Else 
Check for low priority jobs in the queue

Display" Dispatching low priority job in the queue"

\}

\section{PERFORMANCE PARAMETERS OF PER DESTINATION QUEUE}

The Performance metric time is a measure of PDQ's performance which supports the jobs in PDQ. The performance measures are studied using empirical modeling considering the standard parameters of the queuing discipline. Here, we have considered five parameters viz., service time, average waiting time in PDQ, time taken by a job to wait in PDQ, waiting time and average waiting time of a job spent in the system are calculated and the performances of the proposed system are compared with the existing method. The performance parameters are measured as follows, Service time is the time taken by the job to accomplish service at the allotted node and is given by

Service time $=$ sum of total service time of non priority or high priority jobs

Time taken by a job to wait in $P D Q=$ sum of total service time of non priority or priority jobs waiting in $P D Q$ until the task of the job is completed

Waiting time in the system is the time slot between the arrivals for service of a job to till it departs from the system after the completion of job and it can be expressed as

Waiting Time in System = Time between the arrival of the job - departure of job after the execution

\section{RESULTS AND DISCUSSIONS}

Performance analysis of various parameters viz. service time, average waiting time in PDQ, time taken by a job to wait in PDQ, waiting time and average waiting time of a job spent in the system are calculated for the adhoc grid layer and it has been compared with the existing results. Figure 2 shows the variations of service time with number of jobs are depicted for the service time with non-priority based AHGL and priority based m-AHGL. From the figure it is seen that the non-priority method with a steep rise in service time from $222 \mathrm{~ms}-3625 \mathrm{~ms}$, whereas in the proposed priority based system, service time is highly reduced for all the three priority linguistic values. Moreover, the results illustrates that the service time increases gradually with the increase in time for increasing the number of jobs. It is also seen from the figure that for a number of jobs ranging from [25-75], execution time of low priority job is increased. This is due to the presence of high or medium priority jobs in the queue, and since the low priority jobs are given the least preference, service time increases with the increase in the number of jobs ranging from $0-100$. 


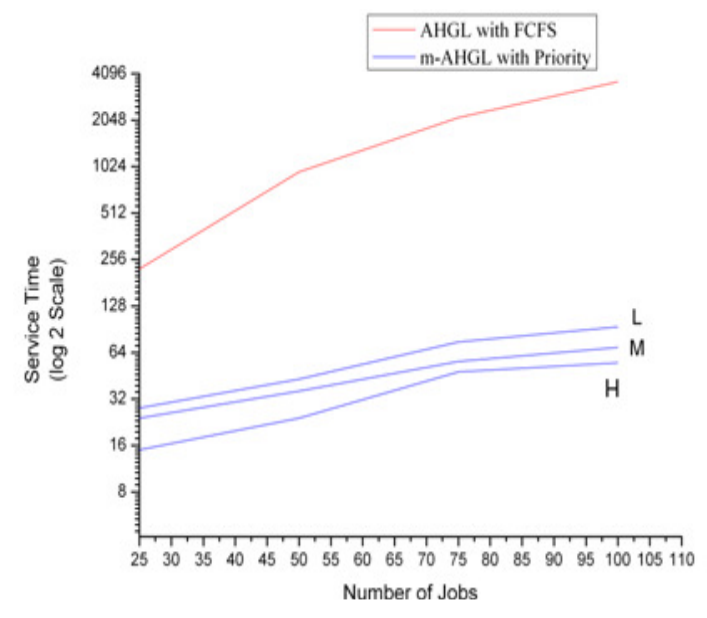

Fig 2.Variation of service time with number of jobs

The variation of waiting time of a job spent in PDQ with number of jobs with low priority, medium priority and high priority is shown in Figure 3. The waiting of the jobs in the existing system with FCFS is $30 \mathrm{~ms}$. The waiting time for the high priority is less than the medium and low priority jobs. The waiting time of the high priority jobs is $3 \mathrm{~ms}$ for the proposed systems which is less than the existing system. This is due to the presence of resourceful nodes, which offers short waiting time, in turn reduces congestion and speed up execution.

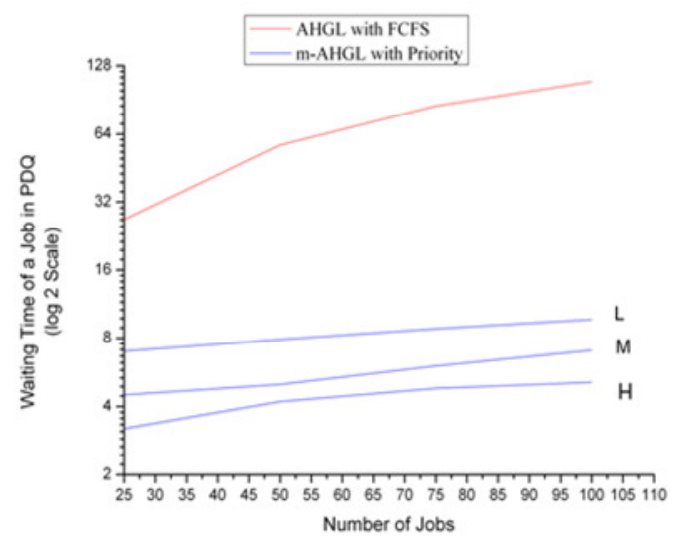

Fig. 3 Variation of waiting time in PDQ time and number of jobs

Variation of time spent by a job in system with the number of jobs spent in the system is shown in Figure 4. In the existing method for number of jobs from 25 to 100, the jobs waiting time is from $260 \mathrm{~ms}$ to $3900 \mathrm{~ms}$. But in the proposed method, waiting time of the prioritized jobs ranges from $14 \mathrm{~ms}$ to $64 \mathrm{~ms}$ respectively for all the low, medium and high priority jobs. The increase in the time spent by the job in the system ranging from 25-50 is due to the arrival of resource consuming of jobs. 


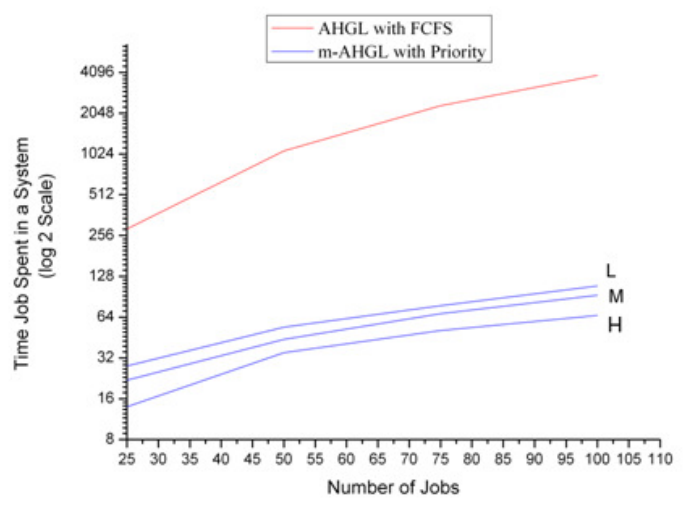

Fig 4.Variation of time a job spent in system and number of jobs

\section{CONCLUSION}

The proposed AHGL with congestion control performs well in terms of service time, waiting time both in the PDQ and in the system. The priority queuing method deployed in the proposed system performs better than the existing AHGL with FCFS algorithm. As only nodes with resources associate to form the ad hoc network, scheduling and dispatching the jobs in this formed network is simple. The nodes with resources are capable of processing the given job in faster way. The scheduling and dispatch of jobs in the formed ad hoc network with resourceful nodes enable faster execution, resulting in more number of jobs to be processed in lesser time. The low priority jobs are assigned with less importance, resulting in an increase in the execution time of the jobs. Our future work is to reduce the execution time of the low priority jobs would result even better performance.

\section{REFERENCES}

[1] Aksetnti Granarov, Bekim Cilku, Igor Miskovski, Sonja Filipska and Dimitar Trajanov: Grid computing implementation in Ad hoc Networks", Advances in Computer and Information Sciences and Engineering, Springer, 196-201, 2008.

[2] Zhoqun Li, Lingfen Sun, Emmanuel C, Ifeachor.: Challenges of Mobile Ad Hoc grids and their applications in E-Health care, in the Proceedings of (CIMED 2005) -2005.

[3] Andreas Pitsillides, Ahmet Sekercioglu.: Fuzzy logic based congestion control”, in Computational Intelligence in Telecommunication networks, pp: 110-154, 2001

[4] W.Ritha and Lilly Robert.: Fuzzy Queues with priority Discipline, in the Journal of Applied Mathematical Sciences, Vol.4, no. 12, pp-575-582, 2010

[5] Yongho Seok, Jaewoo Park and Yanghee Choi.: Queue management algorithm for multi-rate wireless local area networks. $14^{\text {th }}$ IEEE Proc. On Personal, Indoor and Mobile Radio Communications, Vol.3, pp. 2003-2008, 2003.

[6] Umut Akyol, Matthew Andrews, Piyush Gupta, John Honny, Iraj Saniee and Alexander Stolyar, "Joint scheduling and congestion control in mobile Ad Hoc Networks", Proc. IEEE INFOCOM , pp. 619-627,2008.

[7] Krzysztof Kurowski et.al. "Scheduling Jobs on the Grid - Multi criteria approach", Computational methods in science and technology", 12(2) pp. 123-138, 2006.

[8] C.Ernemann, V.Hamscher, U.Schwiegelshohn, R.Yahyapour, A Streit, "On advantages of grid computing for parallel job scheduling", Proc of 2nd IEEEACM International Symposium on Cluster Computing and the Grid CCGRID02 pp 39-39 2002. 
[9] Hameed Vahdat-Nejad, Reza Monsefi, Mahmoud Naghibzadeh, “ A new fuzzy algorithm for global job scheduling in multiclusters and Grids", in Proc. of IEEE CIMSA, pp 54-58, 2007.

[10] B.Roy, "MultiCriteria methodology for decision aiding”, Kluwer, 1996.

[11] Krzysztof et.al , "Scheduling Jobs on the Grid - Multicriteria Approach", Journal of Computational Methods in Science and Technology, 12(2), pp.123-128, 2006.

[12] Gaurav Sharma et.al. "Joint congestion control and distributed scheduling for throughput Guarantees in Wireless Networks", Proc., of IEEE INFOCOMM, pp- 20722080, 2007.

[13] C.Fayad et.al., " Fuzzy grid scheduling using tabu search”, in the Proc. of FUZZ-IEEE 2007, pp 1-6, 2007.

[14] Alexander Folling et.al, “ Robust load delegation in service grid environments”, in IEEE Transactions on Parallel and Distributed Systems, 21(9), pp 1304-1315, 2010.

[15] T.J.Ross,: Fuzzy logic for engineering applications, John Wiley, 1998.

\section{Author Biography}

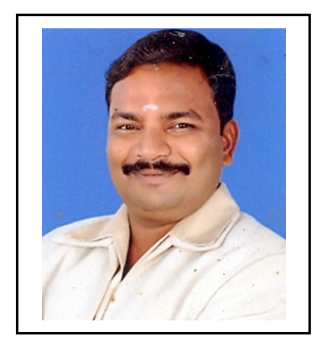

R.Bhaskaran is Associate Professor in the Department of Information Technology, at PSNACET. He is pursuing his currently pursuing his Ph.D., from Anna University Coimbatore. His research area includes ad hoc grid and wireless networks.

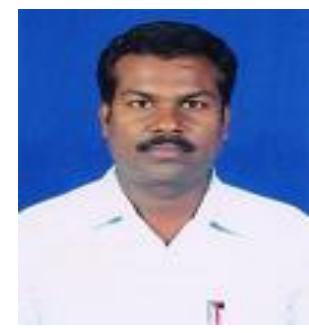

V.Parthasarathyreceived the B.E degree in Electrical and Electronics Engineering from Government College of Technology, Bharathiyar University, Coimbatore, India in 1995. The Master of Engineering degree in Computer Science and Engineering from College of Engineering Guindy, Anna university Chennai in 2004 and Ph.D degree under the faculty of Information and Communication Engineering at Anna University, Chennai in the year 2010. He had published more than 15 papers in referred National and International conferences/Journals. Five students have registered under him for PhD work. His area of interest is optical network. 